\title{
Do blood plasma levels of oxytocin moderate the effect of nasally administered oxytocin on social orienting in high-functioning male adults with autism spectrum disorder?
}

\author{
Monika Althaus ${ }^{1}$ - Yvonne Groen ${ }^{2}$ - Albertus A.Wijers ${ }^{3}$ - Henriette Noltes ${ }^{4}$. \\ Oliver Tucha $^{2} \cdot$ Fred C. Sweep $^{5} \cdot$ Federica Calcagnoli $^{6} \cdot$ Pieter J. Hoekstra $^{1}$
}

Received: 13 April 2016 / Accepted: 4 May 2016 /Published online: 2 June 2016

(C) The Author(s) 2016. This article is published with open access at Springerlink.com

\begin{abstract}
Objective The study investigated whether baseline plasma oxytocin (OXT) concentrations might moderate the effects of nasally administered OXT on social orienting.

Methods Thirty-one males with Autism spectrum disorder (ASD) and thirty healthy males participated in a doubleblind placebo-controlled crossover trial. After administration of the compound, participants were viewing pictures from the International Affective Picture System that represented a systematic variation of pleasant, unpleasant and neutral scenes with and without humans. The outcome measures were a cardiac evoked response (ECR) and a cortical evoked long latency parietal positivity (LPP).

Results Males with ASD had significantly higher plasma baseline levels than the controls. In the absence of general
\end{abstract}

Monika Althaus

m.althaus@accare.nl

1 Department of Child- and Adolescent Psychiatry, University of Groningen, University Medical Center Groningen, Hanzeplein 1, 9713 GZ Groningen, The Netherlands

2 Department of Clinical and Developmental Neuropsychology, University of Groningen, Grote Kruisstraat 2/1, 9712 TS Groningen, The Netherlands

3 Department of Experimental and Work Psychology, University of Groningen, Grote Kruisstraat 2/1, 9712

TS Groningen, The Netherlands

4 Lentis, Autism Team of the North of the Netherlands (ATN), Laan Corpus den Hoorn 102-2, 9728 JR Groningen, The Netherlands

5 Department of Laboratory Medicine, Radboud University Medical Center, Geert Grooteplein 8, 6525 GA Nijmegen, The Netherlands

6 Department of Behavioral Physiology, University of Groningen, Nijenborg 7, 9747 AG Groningen, The Netherlands treatment effects, higher baseline concentrations were found to be associated with larger treatment effects, particularly in the group of males with ASD. Higher post-treatment plasma OXT concentrations were found to be associated with smaller treatment effects and larger orienting responses in the placebo situation in the group of controls.

Conclusions We interpret our findings as suggesting that it is the central availability of OXT determining how much of the nasally administered OXT will become centrally absorbed and how much of it will become released into the bloodstream.

Keywords Baseline plasma oxytocin · Post-oxytocin treatment plasma oxytocin - Social orienting · Autism spectrum disorder $\cdot$ Electrocortical and cardiac evoked responses

\section{Introduction}

Following the many studies on the role of oxytocin (OXT) in prosocial behaviour and attachment (see Bartz et al. 2011; Guastella and MacLeod 2012; Bakermans-Kranenburg and van I Jzendoorn 2013 for extensive reviews), OXT has increasingly been investigated for its role in the pathophysiology and potential treatment of the core deficits in autism spectrum disorders (ASD), especially since its nasal administration has suggested some promising results (Andari et al. 2010; Guastella et al. 2010). ASDs are characterized by deficits in social communication and interaction as well as by restricted, repetitive behaviours and interests (American Psychiatric Association 2013).

In their review on 'the role of oxytocin in psychiatric disorders', Cochran et al. (2013) highlighted three lines of investigation on which studies into the social effects of OXT have 
focused: (1) studies of endogenous peripheral (blood plasma, urinary and salivary) and/or central (cerobrospinal fluid [CSF]) OXT levels, (2) genetic studies and (3) exogenous OXT administration studies. As the present study aimed at investigating whether baseline OXT plasma levels are associated with the effect of nasally administered OXT on social orienting in healthy male adults and male adults with ASD, we have briefly summarized the main findings of the OXT treatment studies conducted on people with ASD as well as the main findings of studies investigating endogenous peripheral and central levels of OXT in a variety of clinical and nonclinical groups.

The first randomized placebo-controlled studies on the acute effects of exogenously administered OXT on the behaviour of individuals with ASD were those by Hollander and colleagues $(2003$; 2007) who administered OXT by intravenous infusion to adults with autism and Asperger's disorder. The experiments showed a greater reduction in repetitive behaviours during OXT infusion compared to placebo (2003) and suggested improvements in affective speech comprehension (2007). In a subsequent study, Guastella et al. (2010) administered OXT nasally to 12- to 19-year-old males with autism or Asperger's disorder and demonstrated improved performance on the Reading the Mind in the-Eyes test (RMET, Baron-Cohen et al 2001) that measures the ability to infer the mood of a person from pictures where only the eye regions of faces are shown. Furthermore, increased cooperative interaction and self-reported feelings of trust were found after nasal administration in young adults with highfunctioning autism and Asperger's disorder when playing a computerized ball throwing game (Andari et al. 2010). These improvements in social behaviour refer to capacities of making inferences about another person's mood or intention, i.e. to capacities that are related to having a theory of the other's mind (ToM), the main ingredient for showing cognitive empathy (e.g. Shamai-Tsoory 2011). In the study by Andari and colleagues, moreover, increased fixation time was found on the eye region of faces in pictures that had to be judged for gender and gaze direction. Interestingly, this response to OXT depended on the patients' social interaction style. Most of the patients who actively displayed approach behaviour, though in an inappropriate or one-sided way, showed positive effects, while most of the individuals who avoided or actively rejected proximity with others showed no response to the treatment. This indicated that the response to OXT may differ for subgroups of patients with ASD. Two longer-term trials have been conducted administering OXT nasally twice daily for 6 weeks (Anagnostou et al. 2012) and once daily for four consecutive days (Dadds et al. 2014) to adults and children with ASD, respectively. Both studies found no significant change in the primary outcome measures of general social functioning and repetitive behaviours, i.e. the core features of ASD. Although the first study found improvements in emotion recognition as measured with the RMET as well as in a broad measure of quality of life, there were no such findings in the second study. The main conclusion by Dadds and colleagues was therefore to be cautious in recommending nasal OXT as a general treatment for young people with autism.

This suggestion was corroborated by the findings of our own study on the effects of nasally administered OXT on social orienting in male adults with ASD (Althaus et al. 2015). In the first place, this study revealed no significant treatment effects, neither in the group of healthy control males nor in the group of males with ASD. However, moderator analysis demonstrated that OXT did enhance social orienting in male adults with ASD who are easily distressed when seeing others in stressful situations, and in healthy males who are highly sensitive to anticipated punishment and criticism or have a low drive for goal achievement. We concluded that individual differences in stress-related avoidance tendencies should be taken into account when considering OXT as a treatment of social deficiencies in autism, which was in agreement with what Dadds et al. (2014), Bartz et al. (2011), and Andari et al. (2010) had previously pointed to, i.e. the person and context dependency that might explain the inconsistencies found in reported OXT effects.

Another moderator of OXT administration effects not yet investigated as such might be the natural variation in endogenous OXT levels. These natural variations are most often measured in a peripheral medium such as blood plasma, urine or saliva, given the discomfort and risks associated with taking CSF by lumbar puncture. Hence, studies of CSF-OXT in humans are scarce and therefore still little is known about the relationship between the central and peripheral availability and action of OXT.

Increased plasma and saliva levels of OXT have repeatedly been found to be related to prosocial behaviour and hence have been suggested to reflect central OXT effects. For example, peripheral OXT levels were elevated in mothers after interacting with their infants (Strathearn et al. 2009). Also, higher plasma OXT levels during pregnancy in primaparous women predicted higher quality of postpartum maternal bonding behaviour (Feldman et al. 2007), while lower levels were predictive of postnatal depression (Skrundz et al. 2011). In the same line, lowered CSF-OXT levels were found in adult women who had been exposed to childhood maltreatment (Heim et al. 2009), and lowered urinary OXT levels were found in children placed in orphanages shortly after birth (Fries et al. 2005). Also in patients with schizophrenia, plasma OXT levels were found to be lower than in healthy subjects, while, in addition, lower levels were associated with more psychotic symptoms (Rubin et al. 2010).

However, the relationship between peripheral OXT levels and social function in ASD is not as straightforward as the above studies would suggest. That is, while lower mean plasma OXT levels were found in 6- to 11-year-old boys with 
autism compared to age matched healthy controls, elevated OXT levels were associated with lower scores on the Vineland Adaptive Behaviour Scale (VABS), which was reverse in the control group (Modahl et al. 1998; Green et al. 2001). This suggests that (high) OXT plasma levels are differentially related to centrally controlled behaviour in autistic vs. healthy children. Yet, in contrast to what has been found for children with ASD, in a study comparing adults with ASD to healthy control adults, baseline blood plasma levels were shown to be significantly higher in the ASD group. In this study, no correlations between plasma OXT levels and impairments in social interactive behaviour were found (Jansen et al 2006). Different from the studies with children, however, the adults were all high functioning while intellectual functioning in the studies of children varied among the participants. This suggests that the OXT system of individuals with ASD may change during lifespan and that OXT plasma levels in ASD may not only be related to developmental but also to intellectual determinants (Jansen et al. 2006).

In summary, the majority of studies suggest that high plasma OXT baseline levels are associated with prosocial skills and behaviour. With regard to the moderating effects of plasma OXT baseline levels on the effect of exogenously administered OXT on social behaviour, this might mean that individuals with lower baseline levels would benefit more from OXT administration and hence show larger treatment effects than those with higher baseline levels.

The present study used the data of our abovementioned previous study showing that nasally administered OXT enhances social orienting in a selective group of male adults with ASD (Althaus et al. 2015). While our previous study took into account the OXT effect-moderating influences of personal characteristics as assessed by questionnaires, the present study investigated whether the baseline level of blood plasma OXT influences the effect of nasally administered OXT on social orienting. As in our previous study, social orienting was investigated by comparing neurophysiological responses to affective pictures with and without social relevance. These responses were an evoked cardiac response (ECR, i.e. stimulusdependent heart rate slowing) and an event-related potential (ERP) derived from the electroencephalogram (EEG), i.e. a late long lasting parietal positivity (LPP). Both types of responses, in particular to the pictures with humans, have previously been shown to discriminate between men and women (Proverbio et al. 2009; Groen et al. 2013; Althaus et al. 2014) and to correlate with empathy-related questionnaire measures (Groen et al. 2013; Althaus et al. 2014).

In agreement with the study by Jansen et al. (2006), we expected that higher blood plasma OXT baseline levels would be found in the group of male adults with ASD than in the group of male controls. Furthermore, we explored whether blood plasma OXT levels correlated with behaviour characteristics as assessed by means of a variety of questionnaires.
We finally expected that males with lower blood plasma OXT baseline levels would benefit more from exogenous OXT administration and hence would show larger treatment effects. This would be reflected by an enhancement of the ECR and LPP responses to affective pictures with humans as compared to pictures without humans after OXT intake. As OXT plasma levels might be differentially related to centrally controlled behaviour in autistic and healthy people (see Modahl et al. 1998; Green et al. 2001; and Jansen et al. 2006), we explored whether the potentially moderating effects might differ for the male adults with ASD and the healthy male adults by investigating these effects in both groups separately.

\section{Methods and material}

The study had been approved by the Medical Ethics Committee of the University Medical Center Groningen, and written informed consent was obtained from all participants.

Most parts of the methods and material have been described in more detail previously (Althaus et al. 2015). Yet, the parts most essential to the present paper have been outlined again below, completed by the description of OXT extraction and analysis.

\section{Participants}

In total, there were $n=61$ participants in the age of $18-34$ years $(\mathrm{M}=22.67 ; \mathrm{SD}=4.22)$. The patient group consisted of $n=31$ males with a Diagnostic and Statistical Manual of Mental Disorders (DSM)-IV diagnosis of ASD, and the control group included $n=30$ healthy male participants. The healthy participants were recruited by advertisements at schools and public spaces. They all reported to be free from psychopathology, which was confirmed by normal scores on the 90 -item Symptom Checklist (SCL-90, Arrindell and Ettema 1986). The patients were recruited via our own outpatient clinic of the University Center Child and Adolescent Psychiatry, Accare, and via the Autism Team of the North of the Netherlands (ATN). The inclusion criterion for the ASD patient group was the presence of a DSM-IV-TR diagnosis in the autistic spectrum, i.e. autistic disorder, Asperger's disorder or pervasive developmental disorder not otherwise specified (PDD-NOS, American Psychiatric Association 2004). For confirmation of the diagnosis established by a well-trained psychiatrist, the patient needed to have a score at or above the cutoff criterion for ASD on at least one of four standardized measures: the Communication or Social Interaction scale of the Autism Diagnostic Observation Scale (ADOS, Lord et al. 2000), which had been assessed in part of the sample as part of routine clinical care; the Social Responsiveness Scale for Adults (SRS-A, Constantino et al. 2003), which was completed by a good acquaintance of the participant; or the autism questionnaire (AQ, Baron-Cohen and Wheelright 2001) completed 
by the participant himself. Further inclusion criteria applying to all participants were IQ $\geq 80$ as measured by the Groningen Intelligence Test (GIT-2, Luteijn and Barelds 2005); normal or corrected to normal vision, and being free from a nasal congestion due to a cold or allergy at the day of testing.

Exclusion criteria for all participants were regular use of psychotropic medication or having a history of alcohol or drug abuse. All participants had to abstain from taking alcohol, stimulants, ecstasy or soft drugs for $20 \mathrm{~h}$, from caffeine for $4 \mathrm{~h}$ and nicotine for $2 \mathrm{~h}$ before the experiment.

The groups did not differ in age and intelligence. Furthermore, participants completed a set of questionnaires on autistic traits, empathic and social skills that were used for correlational analyses (see section 2.5.3).

\section{Procedure}

Participants were invited for the experiment on two different days, separated by 1 week. During the experiment, participants were asked to view a series of photographs (see section 2.3) while their EEG and ECG were recorded. On one of the 2 days, participants received a nasal spray with OXT, 24 IU Syntocinon ${ }^{\circledR}$ (Sigma Tau), while on the other day, the nasal spray contained placebo, a saline solution $(8 \mathrm{mg} / \mathrm{ml}$; Pharmachemie, Haarlem, The Netherlands). The order of the treatments was double-blind and randomized within the groups. The experiment always took part at the same time of the day; either participants started at about 9 am ( $n=15$ controls and $n=11$ males with ASD) or at about $2 \mathrm{pm}(n=14$ controls and $n=20$ males with ASD).

The procedure followed the time schedule depicted in Fig. 1. Participants started with a check on the rules of abstinence and a brief questionnaire on state anxiety (Spielberger's State-Trait Anxiety Inventory, STAI; Spielberger 1973). This was followed by a first venipuncture, with the tube being set on ice and immediately brought to the laboratory. Then the nasal spray was administered. After the experimenter primed the spray, the participant self-administered the spray by alternatingly puffing three times in each nostril (with 4 IU Syntocinon per puff this resulted in 24 IU in total). Participants were instructed to close one nostril and to 'sniff- like' inhale the puff in the other nostril. After spraying, the EEG and ECG equipment was applied by the experimenter (30 $\mathrm{min})$, and a practice block of viewing pictures was performed by the participant $(5 \mathrm{~min})$. This was followed by a second venipuncture, with the period between spraying and the venipuncture lasting about $50 \mathrm{~min}(\mathrm{M}=53.13 \mathrm{~min}$; $\mathrm{SD}=4.92$ ). The viewing of pictures started about $10 \mathrm{~min}$ later, and lasted for $45 \mathrm{~min}$. The period between spraying and starting the task lasted about $60 \mathrm{~min}(\mathrm{M}=60.33$; $\mathrm{SD}=5.48)$. After task completion, the EEG/ECG equipment was taken off, and the participants completed some brief questionnaires about potential side effects of the nasal spray, their experience and motivation during the task, as well as their state of comfort/anxiety (STAI).

\section{IAPS Task}

Participants watched a series of 414 photographs that had been selected from the International Affective Picture System (IAPS) (Lang et al. 2008), representing the following conditions: neutral, positive or negative emotions in scenes with humans (socially relevant conditions); or neutral, positive or negative emotions in scenes without humans (socially irrelevant conditions, see Groen et al. (2013) or Althaus et al. (2014, 2015)). By using E-prime 2.0, the pictures were serially presented for $1 \mathrm{~s}$ with a variable inter-stimulus interval of 3 to $5 \mathrm{~s}$ and required no response. The conditions were balanced and presented in random order. To ensure attentive watching, participants were instructed to press a button whenever a redwhite checkerboard appeared on the screen. These target stimuli appeared randomly in a target/picture ratio of 1:6. Picture presentation was divided into three blocks of each lasting about $13.5 \mathrm{~min}$, separated by short breaks of a few minutes.

\section{Data collection, preprocessing and dependent variables}

\section{Blood sampling and plasma OXT analysis}

Blood samples were obtained using $10 \mathrm{ml}$ EDTA tubes that were immediately put on ice and centrifuged at $1500 \mathrm{~g}$ for 15 min within 1 hour after collection. To separate OXT from
Fig. 1 Time schedule of the experiment

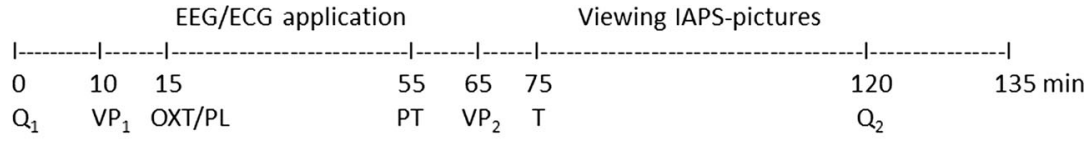

$\mathrm{Q}_{1}$ : Questionnaire on abstinence rules and STAI state

$\mathrm{Q}_{2}$ : Questionnaires on side effects and motivation, STAl state

VP: Venipuncture

PT: Practice Task

EEG/ECG: application of measurement equipment

OXT: OXT administration

PL: Placebo administration

T: IAPS task picture viewing 
its binding proteins, $300 \mu \mathrm{l} \mathrm{HCl}(0.5 \mathrm{M})$ was added to $1 \mathrm{ml}$ of EDTA plasma, and the mixture was prepurified by means of Oasis HLB $(1 \mathrm{ml}, 30 \mathrm{mg})$ extraction cartridges. OXT was eluted from the cartridges by $100 \%$ methanol. The eluates were evaporated in a Savant SpeedVac SC200, and $250 \mu \mathrm{l}$ phosphate buffer $\left(\mathrm{Na}_{2} \mathrm{HPO}_{4} \cdot{ }_{2} \mathrm{H}_{2} \mathrm{O}\right.$ containing $13 \mathrm{mM}$ EDTA, $0.02 \%$ sodiumazide, $0.25 \%$ BSA, $0.1 \%$ Triton X$100, \mathrm{pH} 7.4$ ) was added to the tubes. OXT was quantified by an in-house radioimmunoassay (RIA). In short, $50 \mu \mathrm{l}$ polyclonal antibody (diluted 1:160,000) raised in rabbit was added to $100 \mu \mathrm{l}$ reconstituted sample and pre-incubated at $4{ }^{\circ} \mathrm{C}$ for $72 \mathrm{~h}$. Then, $25 \mu \mathrm{l}$ of ${ }^{125}$ I-labelled OXT (3000 dpm) was added, and incubation was continued for $24 \mathrm{~h}$. Bound and free OXT were separated by a second antibody (10\% sheep antirabbit IgG and $0.01 \%$ rabbit $\operatorname{IgG}$ (carrier protein)/ polyethyleneglycol 6000 solution. Radioactivity in the tubes (bound fraction) was counted using an automatic gammacounter (1470 Wizard TM Wallac, Turku, Finland). Data were analysed with a four parameter fit program.

Recovery was determined by addition of synthetic OXT (Sigma-Aldrich, cat. Nr O-6379) to plasma pools with 1.4 and $2.9 \mathrm{pmol} / 1$ endogenous OXT. The average recovery at these two levels $(n=5)$ was $78 \pm 6 \%$. Within- and betweenassay CVs were 11.1 and $12.8 \%$ at $6.3 \mathrm{pmol} / \mathrm{l}$. All samples were measured in duplo. The synthetic OXT preparation was used for radioiodination and for constructing a dose-response curve. The RIA specifically detects OXT. Cross-reactivity with arginine vasopressin, lysine-vasopressin and desamino$\mathrm{D}$-arginine-vasopressin all were $<0.01 \%$ on mass basis. The analytical sensitivity was $1.5 \mathrm{pmol} / \mathrm{l}$. For statistical analysis of the data, levels below the analytical sensitivity of the assay were assigned a value of $50 \%$ of this sensitivity.

\section{ECG recording and pre-processing of the ECR}

The participants' ECG was recorded from pre-cordial leads by BrainRecorder (brain products) with a sample rate of $500 \mathrm{~Hz}$. For analysis of the ECRs, sequential interbeat intervals (IBIs) occurring around each stimulus presentation were extracted from the R-peak series. IBIs were extracted in segments of
$1 \mathrm{~s}$ before stimulus onset to $4.5 \mathrm{~s}$ after stimulus onset, and sampled every $0.5 \mathrm{~s}$. This resulted in 12 IBI values for each stimulus. For each participant, averages were computed for each stimulus condition, with IBI0 referring to the IBI at stimulus onset and with IBI-1 serving as the baseline value (i.e. the IBI value at $1 \mathrm{~s}$ before stimulus onset was subtracted from all successive IBI values). Finally, by using SPSS, for each participant, the maximal cardiac deceleration was determined in the interval from -0.5 to $4 \mathrm{~s}\left(\mathrm{IBI}_{\mathrm{MAX}}\right)$. For the validity of this measure, we refer to Althaus et al. (2014).

\section{EEG recording and pre-processing of the $L P P$}

EEG was also recorded by BrainRecorder from 61 scalp electrodes that were placed according to the 10-20 system by means of a lycra stretch cap (Electro-Cap Center BV). Based on previous work, 34 electrode positions were selected for analysis.

In the placebo condition, the LPP amplitude was maximal over parietal electrode positions in the time window of 400-1000 ms after stimulus onset, see Fig. 2. To determine the electrode positions showing the strongest task effects, we performed repeated measures analyses on the data from the control group with the factors 'hemisphere', emotional 'valence' and presence of 'humans' on successive time intervals of $100 \mathrm{~ms}$. We found that the largest effects of humans, valence and their interaction were present at $\mathrm{Pz}, \mathrm{P} 3$ and P7 in the intervals running from 600 to $1000 \mathrm{~ms}$. Further analyses of the LPP were therefore performed for the Pz, P3 and P7 potentials occurring in the interval running from 600 to $1000 \mathrm{~ms}$.

\section{Statistical analyses}

\section{Group differences in OXT baseline and OXT post-administration levels}

As the two blood plasma values obtained before OXT and placebo administration, respectively, did not differ
Fig. 2 Topographical maps of the difference waves between pictures with and without humans in the interval 600-1000 ms after stimulus onset. Maps refer to the control group in the placebo condition and show the activity evoked by watching pictures with humans as compared to pictures without humans
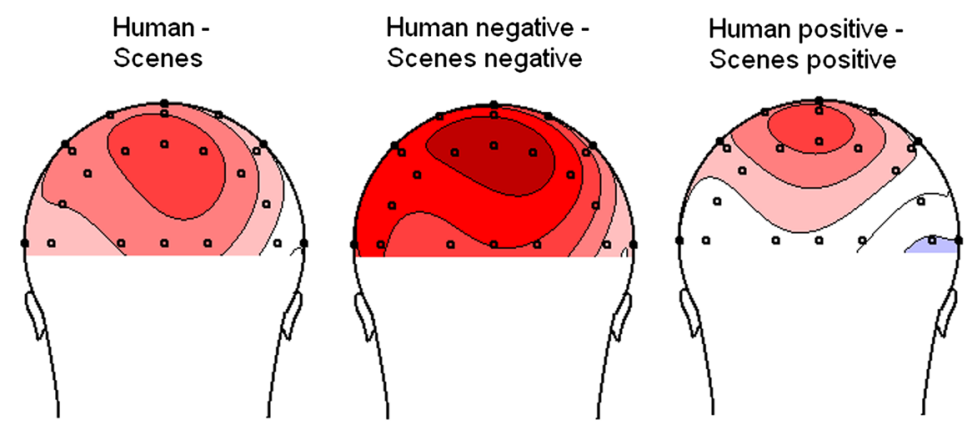

Human neutral Scenes neutral

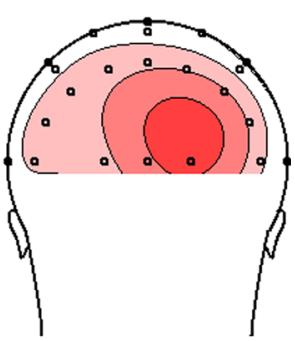

$600 \mathrm{~ms}-1000 \mathrm{~ms}$




from each other, baseline OXT (BLOXT) levels were computed by averaging these two values. This resulted in a more stable measure with less variance. Postadministration levels were adjusted for individual differences in pre-administration levels by subtracting the preadministration values from the post-administration values $(\Delta \mathrm{OXT})$. All values were tested for group differences by means of Student's $t$ tests for independent samples. Differences between pre- and post-administration values were tested by paired Student's $t$ tests.

To check whether the baseline levels (BLOXT) measured in the morning would differ from those that were measured in the afternoon and whether this would differ for the two groups of participants, an ANOVA was carried out with the between subjects (BS) factors 'group' (controls vs. ASD) and 'Time of the Day' (morning vs. afternoon).

\section{Correlations between peripheral OXT levels and behavioural measures}

Pearson's correlations were computed for both BLOXT and $\triangle \mathrm{OXT}$ with all of our behaviour measures including age, intelligence and the questionnaire scores, which are summarized in Table 1.

\section{Treatment moderating effects of OXT baseline level: analyses of co-variance}

Treatment effects on the ERC ( $\left.\mathrm{IBI}_{\mathrm{MAX}}\right)$ and the three LPP values referring to respectively, $\mathrm{Pz}, \mathrm{P} 3$ and $\mathrm{P} 7$ were analysed by a $2 * 2 * 3$ repeated measure ANOVA with the within subject (WS) variables treatment (placebo vs. OXT), human (humans vs. scenes), and valence (neutrals vs. positive vs. negative). To investigate the treatment moderating effect of plasma OXT, the variable BLOXT was entered as a covariate while focussing on the interaction of the covariate with the treatment (by task) effects. Analyses of covariance were carried out both on the whole group and the two groups separately. For effects with valence involved, Greenhouse-Geisser adjusted $p$ values, and the epsilon correction factors were reported. Planned comparisons were carried out by computing contrasts between each of the three valence pairs. All data were controlled for outliers being defined as $\mathrm{z} \leq-3$ and $\mathrm{z} \geq 3$.

For all comparisons, next to the $p$ values, Cohen's $d$ or partial eta squared are presented as measures of effect size. Rejection level was set at $p=.05$, but trend significant $(p \leq .1)$ findings were further elaborated if accompanied by medium or high effect sizes.

\section{Regression analyses and high vs. low plasma OXT group comparisons}

To investigate in which direction OXT effects on the ECR and LPP responses might have been moderated by plasma OXT, we conducted regression analyses of the BLOXT values on the treatment-dependent effect scores. To this end, the effects of (1) human and (2) human*valence were translated into the effect scores (delta values). For their exact computation, we refer to our previous study (Althaus et al. 2015). In a final step (trend), significant regressions, i.e. (trend) significant treatment interactions with the covariate were further elaborated by comparing groups with high vs. low BLOXT values on their orienting responses in both the placebo and OXT condition. This comparison was conducted by a $2 * 2 * 3 * 2$ ANOVA design with the WS variables; treatment, human and valence and the BS variable group formed on the basis of the 33.3th and 66.6th percentiles of the BLOXT values.

Table 1 Relevant behaviour characteristics as measured by questionnaires

\begin{tabular}{llll}
\hline Questionnaire & Authors and year & Scales & Measurement \\
\hline $\begin{array}{l}\text { Autism spectrum quotient (AQ) } \\
\text { Social Responsiveness Scale (SRS) }\end{array}$ & Baron-Cohen et al. 2001 & AQ total & Autistic traits \\
& Constantino et al. 2003 & $\begin{array}{l}\text { Total } \\
\text { Social awareness } \\
\text { Social communication } \\
\text { Social motivation Rigidity }\end{array}$ & Autistic traits \\
& & and repetition & Empathic skills \\
Empathy quotient (EQ) & & EQ total & Empathic skills/ \\
& Baron-Cohen and & personal distress \\
Interpersonal Reactivity & Wheelwright 2004 & Personal distress Emotional & concern Perspective \\
Index (IRI) & Davis 1983 & taking Fantasizing & Avoidance and approach \\
& & BIS & behaviour \\
BIS/BAS questionnaire & Carver and White 1994; Gray & BAS drive & BAS fun \\
& and McNaughton 2000 & BAS reward & State anxiety \\
Spielberger's state-trait & & Trait anxiety & State and trait anxiety \\
anxiety questionnaire (STAI) & Spielberger 1973 & & \\
\hline
\end{tabular}




\section{Results}

Task effects, OXT treatment effects and group differences in task and treatment effects

Both the ECR and LPP were larger in response to pictures with humans and this held for in particular the affective pictures. Yet, our study revealed no group difference and no effects of the nasally administered OXT on the orienting responses. Nor did the groups differ in their treatment response. For the statistical details and corresponding figures presenting the ECR and LPP in placebo and OXT condition, we refer to Althaus et al. (2015).

\section{Group differences in BLOXT levels and correlations of BLOXT levels with behaviour variables}

First of all, morning values of BLOXT did not differ from afternoon values $[F(1,56)=0.16 ; p=.69]$, and this did not differ for the two study groups $[F(1,56)=0.25 ; p=.61]$.

Table 2 summarizes group means and standard deviations of the blood plasma OXT values obtained from the blood samples taken before and after placebo and OXT administration of the control group and the group with ASD, respectively. It shows that the groups differed significantly in their values of the pre-treatment (placebo and OXT) situations with small effect sizes, and in the values averaged across these situations (BLOXT) with moderate effect size. The males with ASD showed significantly higher baseline OXT plasma concentrations than did the control males.

Large differences were found between the OXT levels before and after OXT administration [for the whole group: $t$ $(59)=11.95 ; p<.001 ; d=1.55$; controls: $t(29)=8.26$; $p<.001 ; d=1.51$; ASD: $t(28)=8.67 ; p<.001 ; d=1.61]$, yet groups did not differ in these $\Delta$ OXT levels $\left[F_{\text {Time* Group }}(1\right.$, $57)=.45 ; p=.51]$.

Table 2 Group means and differences in plasma OXT values
BLOXT did not correlate with any of the behaviour measures. It did, however, correlate significantly with the level of intelligence in the whole group $(r=.31 ; n=61 ; p=.02)$ and in the group with $\operatorname{ASD}(r=.38 ; n=31 ; p=.04)$.

\section{Baseline OXT level as moderator of the OXT treatment effect}

We found a significant interaction of BLOXT with the OXT treatment effect on responses to pictures with humans (as compared to the pictures without humans) for the ECR $\left[F_{\text {Treatment*Human*BLOXT }}(1,27)=5.69 ; p=.024 ; \eta^{2}=.17\right]$ and a trend significant interaction with medium effect size for the LPP at $\mathrm{Pz}\left[F_{\text {Treatment*Human*BLOXT }}(1,29)=3.35 ; p=.07\right.$; $\left.\eta^{2}=.10\right]$ in the group with ASD, but not in the control group. However, the two groups did not differ significantly from each other in this BLOXT-dependent response to the treatment [ECR: $F(1,27)=1.22 ; p=.27 ; \eta^{2}=.02 ; \mathrm{LPP}$ at Pz: $F(1$, $\left.59)=2.53 ; p=.12 ; \eta^{2}=.04\right]$. For the LPP at P7, a significant interaction was found for the whole group of participants $\left[F_{\text {Treatment*Human*BLOXT }}(1,59)=3.96 ; p=.04 ; \eta^{2}=.06\right]$.

Regression of the BLOXT values upon the OXT effect on the ECR response to pictures with humans shows that in the group with ASD lower BLOXT values were associated with smaller effects. The same holds for the regression of BLOXT upon the OXT treatment effect on the LPP responses to human pictures (Fig. 3a-c). For none of the orienting responses, BLOXT-moderating effects were found in the placebo situation.

Comparisons of the $33.3 \%$ males $(n=10)$ with ASD showing the lowest BLOXT levels $(\leq 0.90 \mathrm{pmol} / \mathrm{l})$ with the $33.3 \%$ $(n=10)$ males showing the highest BLOXT levels $(\geq 1.74 \mathrm{pmol} / \mathrm{l})$ resulted in a trend significant interaction of this group variable with the treatment effect on the ECR response to human pictures with high effect size $\left[F_{\text {Treatment*Human*BLOXTGroup }}(1,18)=2.99 ; p=.1 ; \eta^{2}=.15\right]$.

\begin{tabular}{llllll}
\hline & Controls $n=30$ & ASD $n=31$ & \multicolumn{2}{l}{ Group differences } & \\
\cline { 4 - 6 } & Mean (sd) & Mean (sd) & t (df) & $p$ & $d$ \\
\hline OXT $^{\text {a } \text { before PL }}$ & $0.66(0.93)$ & $1.50(2.07)$ & $-1.96(59)$ & .05 & 0.39 \\
OXT after PL $^{\mathrm{b}}$ & $0.80(1.33)$ & $0.83(0.95)$ & $-0.89(59)$ & $>.1$ & 0.23 \\
OXT before OXT $^{\mathrm{c}}$ & $0.67(0.94)$ & $1.32(1.67)$ & $-2.37(59)$ & .02 & 0.47 \\
OXT after OXT $^{\mathrm{c}}$ & $9.88(6.34)$ & $9.60(5.04)$ & $0.19(57)^{\mathrm{d}}$ & $>.1$ & 0.05 \\
BLOXT & $0.67(0.77)$ & $1.34(1.05)$ & $-2.85(59)$ & .006 & 0.73 \\
$\Delta$ OXT & $9.20(6.10)$ & $8.22(5.11)$ & $0.67(57)^{\mathrm{d}}$ & $>.1$ & 0.17 \\
\hline
\end{tabular}

${ }^{\text {a }}$ Plasma OXT measured in pmol/1

b, c Placebo/OXT administration

${ }^{\mathrm{d}}$ There were two missing values in the ASD group

BLOXT: mean value of OXT concentrations before placebo and oxytocin administration. $\triangle \mathrm{OXT}$ : OXT after $\mathrm{OXT}^{\mathrm{c}}$ minus OXT before $\mathrm{OXT}^{\mathrm{c}}$ 



Fig. 3 Regression of the baseline OXT concentrations upon the treatment effect on the orienting responses ECR (a), LPP at Pz (b) and LPP at P7 (c) to pictures with humans as compared to pictures without humans in male adults with ASD

For the LPP at Pz, comparison of the two groups revealed a significant treatment by group interaction $\left[F_{\text {Treatment*Human*BLOXTGroup }}(1,18)=4.30 ; p=.05 ; \eta^{2}=.19\right]$, and for the LPP at P7, when comparing the $33.3 \%(n=20)$ participants of the whole group showing the lowest BLOXT values $(\leq 0.49 \mathrm{pmol} / \mathrm{l})$ with the $n=20$ participants showing the highest BLOXT values ( $\geq 1.38 \mathrm{pmol} / \mathrm{l})$, a significant group interaction with the treatment effect was found $\left[F_{\text {Treatment*Human*BLOXTGroup }}(1,38)=6.12 ; p=.02 ; \eta^{2}=.14\right]$. The interactions are plotted in Fig. 4a-c.

Summarizing the moderating influence of baseline OXT on the OXT treatment effects, we found that, opposite to what was expected, males with higher plasma OXT concentrations showed larger OXT treatment effects as expressed by an enhancement of their ECR and LPP responses to pictures with humans during OXT intake as compared to the placebo situation. This was found in particular for the males with ASD. An illustration of this OXT administration-induced enhanced orienting to pictures with humans in only the high baseline OXT group is given in Fig. 5 showing the LPP response at Pz, which discriminated the two groups of ASD participants with, respectively, low and high BLOXT values from each other with high effect size.

\section{Additional analyses}

To obtain more insight into the mechanisms of how peripheral OXT levels may influence OXT administration- induced central effects, we investigated whether plasma OXT levels assessed after nasal OXT administration would be related to the effects of nasal OXT administration on social orienting. If larger peripheral post-administration levels were found to be associated with greater OXT treatment effects, this would suggests a parallel increase of peripheral and central levels after nasal OXT administration. If, however, larger postadministration plasma levels were associated with smaller treatment effects, this might suggests that less OXT had become centrally active due to, for example, leakage, less absorption or central saturation. Analyses were carried out on the baseline-adjusted post-administration levels ( $\triangle \mathrm{OXT}$ ).

Regression of the post-administration values upon the OXT effect on the ECR responses to pictures with humans 
OXT effect dependency on plasma OXT

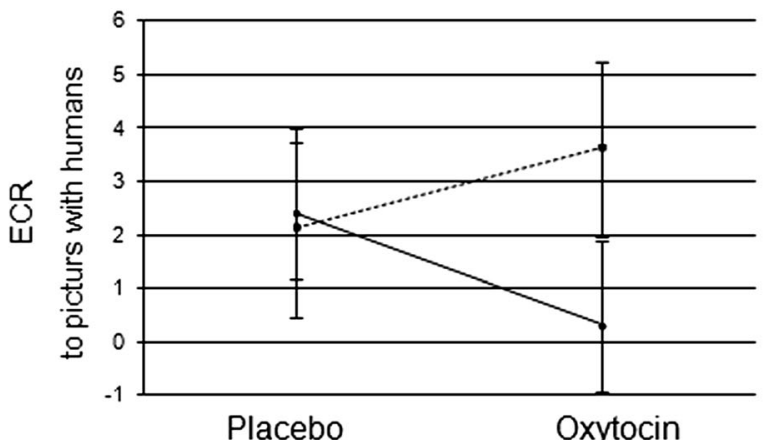

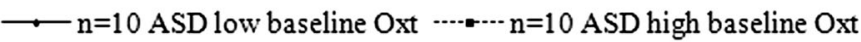

a

OXT effect dependency on plasma OXT

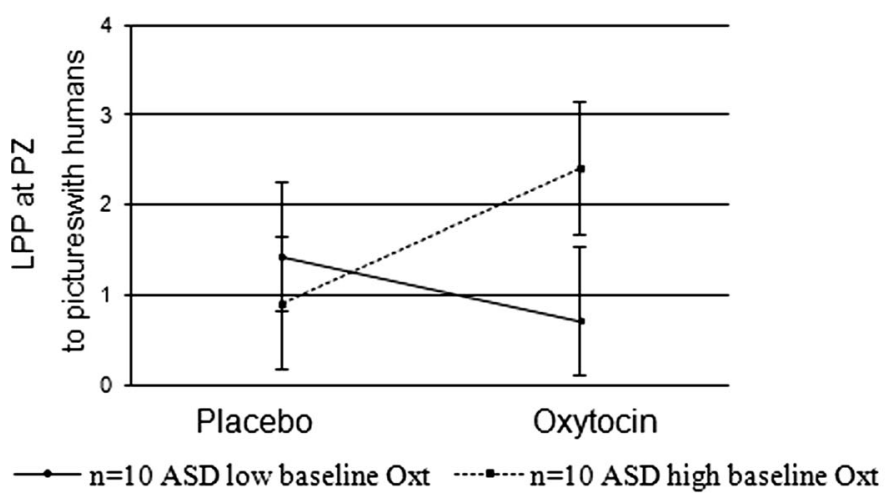

b



$\mathrm{C}$

Fig. 4 Means and SEMs of the human effect illustrating the increase of the orienting responses ECR (a), LPP at Pz (b) and LPP at P7 (c) to pictures with humans in the OXT condition for only the ASD males with high baseline levels of blood plasma OXT

\section{ASD}

low baseline OT values

Placebo

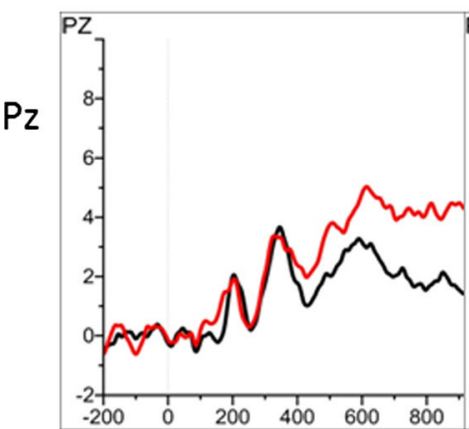

Oxytocin

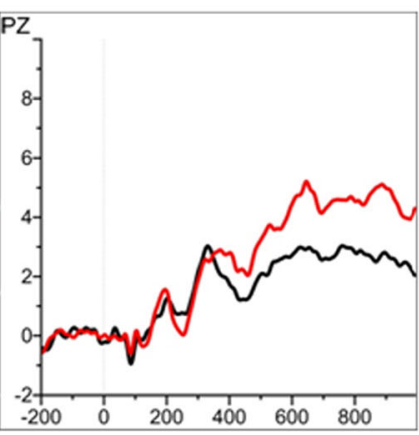

human high baseline OT values

Placebo Oxytocin

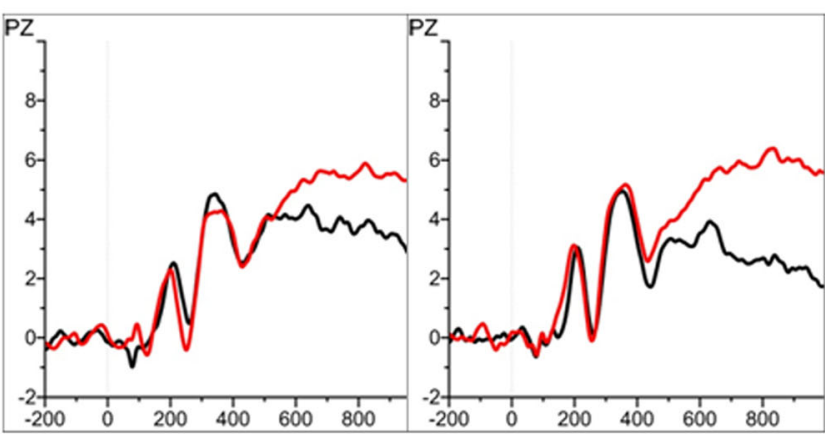

scenes

Fig. 5 ERPs (LPP at Pz) in response to pictures with and without humans of ASD males having low $(\leq \mathrm{P} 33)$ and high $(\geq \mathrm{P} 66)$ blood plasma OXT baseline values 
showed that in the whole group of participants lower $\Delta \mathrm{OXT}$ values were associated with larger effects $(c=6.21, \beta=-.30$; $n=61 ; t=-2.34 ; p=.02)$. The same was found for the regression of the OXT treatment effect on the LPP Pz response to negative human pictures, yet in only the control group $(c=2.10, \beta=-.36 ; n=30 ; t=-2.02 ; p=.05)$ and the regression on the LPP P7 response to human pictures $(c=0.78$, $\beta=-.43 ; n=30 ; t=-2.49 ; p=.02)$ again in only the control group (see Fig. 6a-c). Moreover, a significant positive association was found between $\triangle \mathrm{OXT}$ and the LPP P7 response to pictures with humans in the placebo situation $(c=-0.16$, $\beta=.48 ; n=30 ; t=2.95 ; p=.006$; see Fig. $6 \mathrm{~d}$ ).

Summarizing the association of post-administration peripheral OXT with the OXT treatment effects, we found that individuals with low post-administration values showed an enhancement of their social orienting after OXT intake while those with high post-administration levels showed a decrease. Figure 7a, b illustrates the OXT administration-induced enhanced orienting to pictures with humans as is assessed by

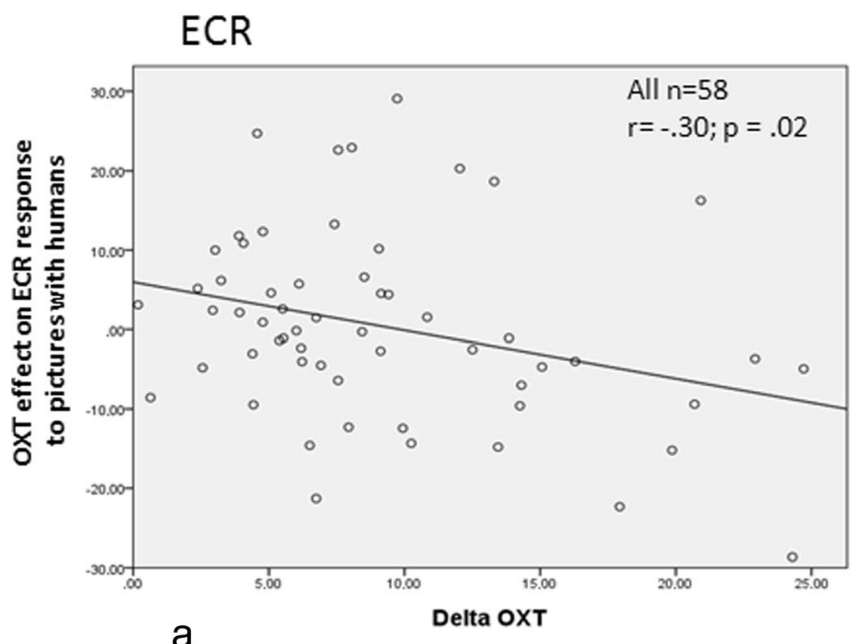

a

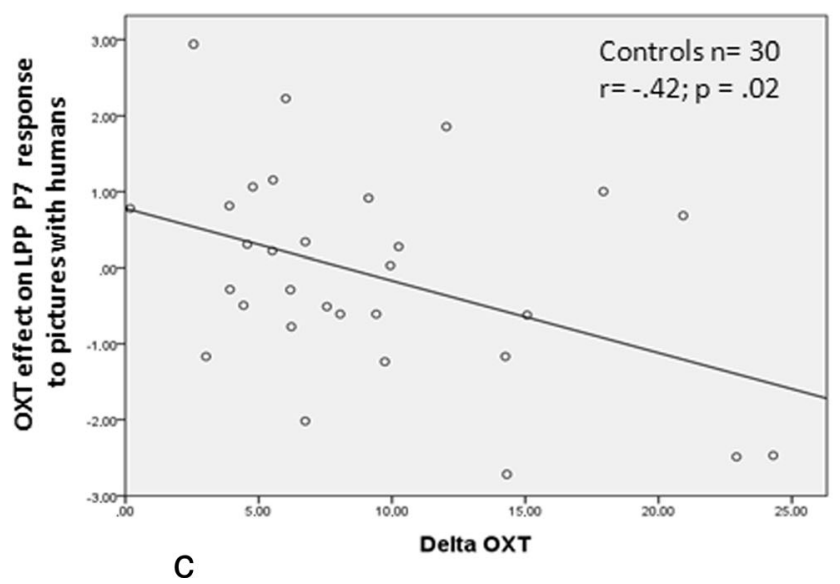

Fig. 6 Regression of the baseline-adjusted post-administration OXT concentrations upon the treatment effects on the orienting responses ECR (a), LPP at Pz (b), LPP at P7 (c) to (negative, the LPP at P7 in only the low level ( $\leq$ P33) postadministration OXT group, which differed significantly from the high level ( $\geq$ P 66 ) post-administration group $\left[F_{\text {Treatment*Human*Valence(negative) }}{ }^{*} \Delta\right.$ OXTGroup $(1,18)=4.70$; $\left.p=.04 ; \eta^{2}=.21\right]$. The figures moreover show the significant difference between the groups in their placebo responses to pictures with humans.

\section{Discussion}

The present study investigated whether OXT treatment effects on social orienting are moderated by blood plasma OXT levels. We first investigated whether there were any correlations between the plasma OXT levels and a variety of personality characteristics and behaviour problems as measured by questionnaires. No significant correlations were found, not even for the previously found OXT effect-moderating personality characteristics that were related to coping with social stress, anticipation



LPP P7

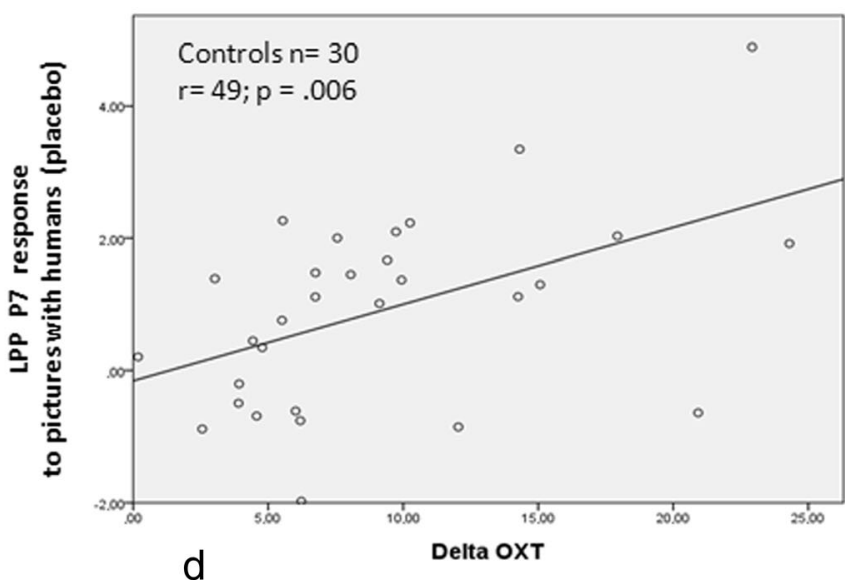

$\mathrm{Pz}$ ) pictures with humans and upon the LPP P7 orienting responses in the placebo situation (d) 


\section{Controls}

low post-administration OXT values

Placebo

Oxytocin

high post-administration OXT values

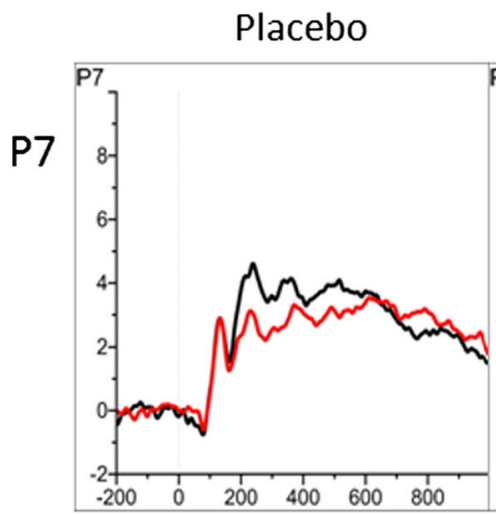

Placebo

Oxytocin


a

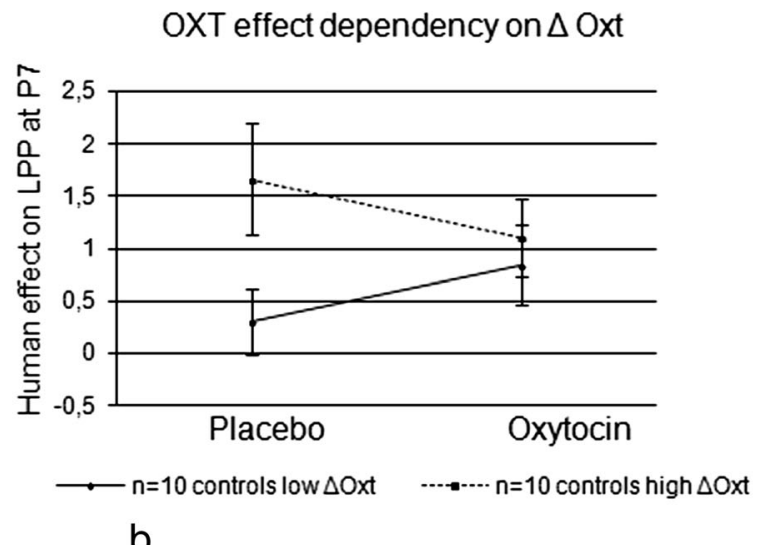

b

Fig. 7 ERPs at P7 in response to pictures with and without humans of the control males having low $(\leq \mathrm{P} 33)$ and high $(\geq \mathrm{P} 66)$ post-administration blood plasma oxytocin values, respectively (a). Means and SEMs of the human effect illustrating the increase of the social orienting response in

the OXT condition for only the males with low levels of postadministration blood plasma OXT. The graph also demonstrates the group differences in the placebo situation (b)

of punishment and criticism or the drive for goal achievement (see Althaus et al. 2015). This corresponds with the findings by Jansen et al. (2006) who also did not find plasma OXT baseline levels to be correlated with any of their measures assessing autism- and anxiety-related behaviour in high-functioning adults with ASD or healthy controls.

Also in agreement with the study by Jansen et al. (2006), yet in contrast to what has been found in children with ASD (Modahl et al. 1998; Green et al 2001), is our finding that our group of ASD male adults had significantly higher baseline plasma OXT levels than our male adult control group. The difference with the findings in children was explained by Jansen and colleagues as being due to their sample consisting of intellectually high-functioning adults with ASD suggesting both developmental and intellectual factors playing a role in the increased OXT plasma levels. The suggestion of intellectual functioning being associated with OXT plasma levels in individuals with ASD is supported by the positive correlation we found between baseline plasma OXT and the level of intelligence in our high-functioning ASD group. This group, though not differing in mean IQ from our control group, showed significantly greater variance (Leven's test: $F=8.14$; $p<.006)$ caused by several very high IQ scores $(n=7$ : IQ $>120$ ). Considering that only the ASD participants with high baseline OXT levels showed an increase in their social orienting responses after OXT administration, and that baseline OXT levels were positively correlated with IQ, we investigated whether intelligence would be a moderator of the OXT administration effect but did not find so. Therefore, IQ seems to be functionally related to the baseline plasma OXT concentrations but not to the effect of exogenously administered OXT on social orienting.

The seemingly counterintuitive finding of male adults with ASD having higher plasma concentrations of OXT corroborates not only the finding by Jansen et al. (2006) but also those of several other studies. Higher plasma concentrations were 
also found in males with a major depression (MD) as compared to females with MD as well as to healthy male controls (Yuen et al. 2014). This gender dependency proves how important it is to take into account sex differences when studying the role of OXT in psychopathological conditions. Plasma concentrations were also shown to positively correlate with fear of romantic attachment in healthy (predominantly female) individuals (Marazziti et al. 2006) and with the severity of social anxiety and dissatisfaction with social relationships in male and female patients with a Generalized Social Anxiety Disorder (GSAD) (Hoge et al. 2008). Higher plasma OXT concentrations are hence not unique to male adults with ASD and are suggested to go along with social anxietyrelated problems.

OXT release into the peripheral circulation, however, may depend on what happens shortly before and/or during blood collection. As both physical and psychological stress have been suggested to activate the hypothalamic-pituitaryadrenocortical (HPA) axis and as neuroendocrine stress responses have been characterized by, among others, OXT release from the pituitary gland into the peripheral circulation (e.g. Onaka 2004), one might argue that the venipunctures were more stressful to our ASD participants than to the control males leading to larger OXT releases into the bloodstream. There are several arguments which may weaken this hypothesis. In the studies by Modahl et al. (1998) and Green et al. (2001), children with ASD were involved showing lower plasma concentrations although they had undergone venipuncture as well. The present study included only participants with ASD who had indicated not to be afraid to undergo two venipunctures. Furthermore, no correlations were found between the decrease in OXT concentration and the decrease in the state anxiety measures obtained in the placebo situation, although the latter was shown to be significant in both groups (Althaus et al. 2015). If the punctures had been more stressful to the males with ASD leading to more peripheral OXT release, we would have expected a significant correlation between the decrease in STAI scores and plasma levels in the placebo situation, especially in the group of males with ASD.

Still more intriguing than the higher OXT baseline level found for the males with ASD is our finding that OXT administration effects on social orienting were moderated by peripheral OXT baseline concentrations. This moderation was opposite to what we had expected according to the literature. Assuming that the reported positive correlations of peripheral OXT levels with centrally controlled prosocial behaviour (e.g. Feldman et al. 2007; Strathearn et al. 2009) and negative correlations with psychopathological symptoms (e.g. Rubin et al. 2010; Skrundz et al. 2011) implicate that plasma OXT concentrations are reflective of intra-cerebral OXT concentrations and activity, we inferred a greater benefit from exogenously administered OXT in individuals with low peripheral levels of OXT. Yet, high levels of baseline plasma OXT were found to be associated with beneficial effects of nasally administered OXT on social orienting, while low levels were accompanied by a decrease in the orienting responses when OXT was administered. Figure 4 shows that the differences between individuals with high and low baseline plasma levels refer to the social orienting responses in only the OXT condition. Our findings therefore suggest that peripheral baseline OXT levels are predictive of the effects of extraneously administered OXT on centrally controlled social orienting but not of social orienting itself.

As the OXT treatment effects turned out to be beneficial in those who have higher baseline peripheral levels, we speculate that a high peripheral level might be indicative of an initially lower central baseline level which is likely to be enhanced by nasal administration of OXT. While the lacking correlations between plasma and CSF levels of OXT reported by Takagi et al. (1985), Winslow et al. (2003), Altemus et al. (2004), and Kagerbauer et al. (2013) have been supposed to reflect dissociated peripheral and central OXT release patterns, the positive correlations between absolute central and blood plasma peak values after nasal administration found in rats by Neumann et al. (2013) suggest the opposite. These findings, however, do not contribute to explain the direction in which we found the plasma baseline levels being associated with the central effects of nasally administered OXT. Might our findings on the associations of the treatment effects with the post-administration level shed some more light on the relationship between plasma OXT levels and the susceptibility to extraneously administered OXT? First, we found a significant and about tenfold increase of the plasma OXT values about $53 \mathrm{~min}( \pm \mathrm{SD}=4.92)$ after nasal administration. Adjusted for pre-administration values, these increases did not differ between our two groups of healthy male adults and male adults with ASD, neither in their absolute values, nor in their values relative to baseline. We further found that individuals with high post-administration OXT plasma levels showed decreased social orienting after OXT intake. This was assumed to be observed if higher peripheral postadministration levels reflect that less OXT had become centrally available or active due to leakage, less absorption or central saturation. Whereas intranasal applications of OXT have been shown to result in very large increases in circulating OXT i.e. to levels far above those that are needed for physiological effects, only small amounts may enter the brain while penetrating some specific brain regions yet not entering the CSF (Leng and Ludwig 2016; Neumann et al 2013). This may be due to leakage that has been suggested indeed to occur as a consequence of intranasal spray delivery. In studies on macaques, comparing nasal spray administration with OXT nebulizer delivery, a significant and similar elevation of CSF OXT levels was found for both delivery methods while plasma OXT was significantly enhanced only after intranasal administration (Dal Monte et al. 2014; Modi et al. 2014). The 
authors explained the significant elevation of plasma OXT by the possible formation of larger droplets from the spray, which would rest on the nasal mucosa and hence provide for vascular absorption as compared to the nebulizer forming a much finer mist and allowing more of the substance to be breathed in deeper towards the epithelium.

Yet, post-administration levels were not only related to the treatment effects (see Fig. 7a, b) but also to the social orienting responses in the placebo situation. The groups with low and high post-administration plasma levels differed in their ECR and LPP P7 responses to pictures with humans in especially the placebo situation (LPP at P7: $t=2.19 ; \mathrm{df}=18 ; p=.04$; ECR: $t=1.8 ; \mathrm{df}=36 ; p=.08$ ), which means that the groups differing in their post-administration OXT plasma levels also differ in their pre-administrative social orienting responses that are centrally controlled because they demand the evaluation of information as being relevant or not. This cannot be explained by differences in leakage of the administered OXT from the nasal mucosa into the bloodstream but might agree with the above proposed hypothesis of individual differences in central absorption being due to differences in central bioavailability. Individuals with lower post-administration plasma concentrations might have had an initially decreased central availability of OXT with the consequence that more of the OXT administered became centrally active while less was released into the peripheral bloodstream.

The study included only males with an IQ $\geq 80$, with seven males of the ASD group having an IQ of even $\geq 120$. This confines generalizability of our findings to a subgroup of only high-functioning males with ASD. As IQ was found to correlate with baseline OXT plasma concentrations in this group, OXT studies with low functioning individuals with ASD are warranted.

The number of participants was too small to enter multiple covariates into the design in order to investigate the potential interactive moderating of OXT effects by OXT baseline levels and the previously found questionnaire-assessed behaviour. Yet, for the behaviour that was previously found to moderate the effects of OXT on social orienting, no correlations were found with OXT baseline levels.

The findings on the OXT moderations cannot be interpreted as unambiguously specific to the two groups investigated, i.e. the findings on baseline OXT plasma concentration as moderating the treatment effect in only the males with ASD and the findings on post-treatment OXT concentrations as moderating the treatment effect in only the control group since group differences were not found to be significant.

Yet, from the findings of the present study, we can conclude that plasma OXT levels are associated with the effects of nasally administered OXT on social orienting as assessed by neurophysiological responses to socially relevant information. As our findings were rather unexpected, their interpretation is a tentative one; we propose that it is the central availability of OXT that determines how much of the nasally administered OXT will become absorbed and centrally active, and how much of it will become released into the bloodstream. Yet, still too little is known about the relationship between peripheral and central OXT concentrations on the one hand and peripheral concentrations and the central action of exogenously administered OXT on the other hand. This holds for healthy individuals as well as for individuals with a psychopathological condition and means that both more preclinical and clinical studies are warranted. Preclinical studies might, for example, compare OXT plasma and CSF or local brain OXT concentrations after both nasal administration and intracerebroventricular (ICV) infusion while at the same time investigating social behaviour. This has, to the best of our knowledge, not yet been done but would be an extension of, for example, the studies by Calcagnoli et al. $(2015 \mathrm{a}, \mathrm{b})$ who compared the effects of ICV with intranasal OXT administration on aggressive and prosocial behaviour in rats but did not analyse plasma OXT concentrations, and by Neumann et al. (2013) who compared changes in central and peripheral OXT concentrations after nasal and intraperitoneal administration but did not relate these concentrations to central effects, i.e. to changes in social behaviour. Clinical studies on the effects of exogenous OXT administration (preferably by nasal spray) should more and more take into account peripheral OXT levels as a potential treatment moderator.

Acknowledgments We give our special thanks to the Accare foundation for the financial support of this investigation. We are further grateful for the kind support by the Autism Team of the North of the Netherlands and the Jonx Team of Lentis, Groningen, in recruiting our participants.

Compliance with ethical standards The study had been approved by the Medical Ethics Committee of the University Medical Center Groningen, and written informed consent was obtained from all participants.

Conflict of interest The authors declare that they have no conflicts of interest.

Open Access This article is distributed under the terms of the Creative Commons Attribution 4.0 International License (http:// creativecommons.org/licenses/by/4.0/), which permits unrestricted use, distribution, and reproduction in any medium, provided you give appropriate credit to the original author(s) and the source, provide a link to the Creative Commons license, and indicate if changes were made.

\section{References}

Altemus M, Fong J, Yang R, Damast S, Luine V, Ferguson D (2004) Changes in cerebrospinal fluid neurochemistry during pregnancy. Biol Psychiatry 56:386-392. doi:10.1016/j.biopsych.2004.06.002

Althaus M, Groen Y, van der Schaft L, Minderaa RB, Tucha O, Mulder LJ, Wijers AA (2014) Sex differences in orienting to pictures with 
and without humans: evidence from the cardiac evoked response (ECR) and the cortical long latency parietal positivity (LPP). PLoS One 9:e108224. doi:10.1371/journal.pone.0108224

Althaus M, Groen Y, Wijers AA, Noltes H, Tucha O, Hoekstra PJ (2015) Oxytocin enhances orienting to social information in a selective group of high-functioning male adults with autism spectrum disorder. Neuropsychologia

American Psychiatric Association (2004) Diagnostic and statistical manual of mental disorders, 4th edn, text revision (DSM-IV-TR). American Psychiatric Press, Washington

American Psychiatric Association (2013) Diagnostic and statistical manual of mental disorders, 5th edn (DSM-5). American Psychiatric Press, Washington

Anagnostou E, Soorya L, Chaplin W, Bartz J, Halpern D, Wasserman S, Wang AT, Pepa L, Tanel N, Kushki A, Hollander E (2012) Intranasal oxytocin versus placebo in the treatment of adults with autism spectrum disorders: a randomized controlled trial. Mol Autism 3:162392-3-16. doi:10.1186/2040-2392-3-16

Andari E, Duhamel J, Zalla T, Herbrecht E, Leboyer M, Sirigu A (2010) Promoting social behavior with oxytocin in high-functioning autism spectrum disorders. Proc Natl Acad Sci U S A 107:4389-4394

Arrindell WA, Ettema JHM (1986) SCL-90: Handleiding bij een multidimensionele psychopathologie indicator. (Manual) test publishers, Lisse, Swets \& Zeitlinger

Bakermans-Kranenburg MJ, van I Jzendoorn MH (2013) Sniffing around oxytocin: review and meta-analyses of trials in healthy and clinical groups with implications for pharmacotherapy. Transl Psychiatry 3: e258. doi:10.1038/tp.2013.34

Baron-Cohen S, Wheelwright S (2001) The autism-spectrum quotient (AQ): evidence from Asperger syndrome/high-functioning autism, males and females, scientists and mathematicians. J Autism Dev Disord 31:5-17

Baron-Cohen S, Wheelwright S (2004) The empathy quotient: an investigation of adults with Asperger syndrome or high functioning autism, and normal sex differences. J Autism Dev Disord 34:163-175

Baron-Cohen S, Wheelwright S, Hill J, Raste Y, Plumb I (2001) The "Reading the Mind in the Eyes" test revised version: a study with normal adults, and adults with Asperger syndrome or highfunctioning autism. J Child Psychol Psychiatry 42:241-251

Bartz JA, Zaki J, Bolger N, Ochsner KN (2011) Social effects of oxytocin in humans: context and person matter. Trends Cogn Sci (Regul Ed) 15:301-309. doi:10.1016/j.tics.2011.05.002

Calcagnoli F, Kreutzmann JC, de Boer SF, Althaus M, Koolhaas JM (2015a) Acute and repeated intranasal oxytocin administration exerts anti-aggressive and pro-affiliative effects in male rats. Psychoneuroendocrinology 51:112-121. doi:10.1016/j.psyneuen. 2014.09.019

Calcagnoli F, Stubbendorff C, Meyer N, de Boer SF, Althaus M, Koolhaas JM (2015b) Oxytocin microinjected into the central amygdaloid nuclei exerts anti-aggressive effects in male rats. Neuropharmacology 90:74-81. doi:10.1016/j.neuropharm.2014. 11.012

Carver CS, White TL (1994) Behavioral inhibition, behavioral activation, and affective responses to impending reward and punishment: the BIS/BAS Scales. J Pers Soc Psychol 67:319-333. doi:10.1037/ 0022-3514.67.2.319

Cochran DM, Fallon D, Hill M, Frazier JA (2013) The role of oxytocin in psychiatric disorders: a review of biological and therapeutic research findings. Harv Rev Psychiatry 21:219-247. doi:10.1097/HRP. 0b013e3182a75b7d

Constantino JN, Davis SA, Todd RD, Schindler MK, Gross MM, Brophy SL, Metzger LM, Shoushtari CS, Splinter R, Reich W (2003) Validation of a brief quantitative measure of autistic traits: comparison of the social responsiveness scale with the autism diagnostic interview-revised. J Autism Dev Disord 33:427-433
Dadds MR, MacDonald E, Cauchi A, Williams K, Levy F, Brennan J (2014) Nasal oxytocin for social deficits in childhood autism: a randomized controlled trial. J Autism Dev Disord 44:521-531. doi:10.1007/s10803-013-1899-3

Dal Monte O, Noble PL, Turchi J, Cummins A, Averbeck BB (2014) CSF and blood oxytocin concentration changes following intranasal delivery in macaque. PLoS One 9:e103677. doi:10.1371/journal.pone. 0103677

Davis M (1983) Measuring individual-differences in empathy-evidence for a multidimensional approach. J Pers Soc Psychol 44:113-126. doi:10.1037//0022-3514.44.1.113

Feldman R, Weller A, Zagoory-Sharon O, Levine A (2007) Evidence for a neuroendocrinological foundation of human affiliation: plasma oxytocin levels across pregnancy and the postpartum period predict mother-infant bonding. Psychol Sci 18:965-970

Fries AB, Ziegler TE, Kurian JR, Jacoris S, Pollak SD (2005) Early experience in humans is associated with changes in neuropeptides critical for regulating social behavior. Proc Natl Acad Sci U S A 102: $17237-17240$

Gray JA, McNaughton N (eds) (2000) The neuropsychology of anxiety: an enquiry into the functions of the sept-hippocampal system. Oxford University Press, Oxford

Green L, Fein D, Modahl C, Feinstein C, Waterhouse L, Morris M (2001) Oxytocin and autistic disorder: alterations in peptide forms. Biol Psychiatry 50:609-613

Groen Y, Wijers AA, Tucha O, Althaus M (2013) Are there sex differences in ERPs related to processing empathy-evoking pictures? Neuropsychologia 51:142-155. doi:10.1016/j.neuropsychologia. 2012.11.012

Guastella AJ, MacLeod C (2012) A critical review of the influence of oxytocin nasal spray on social cognition in humans: evidence and future directions. Horm Behav 61:410-418. doi:10.1016/j.yhbeh. 2012.01.002

Guastella AJ, Einfeld SL, Gray KM, Rinehart NJ, Tonge BJ, Lambert TJ, Hickie IB (2010) Intranasal oxytocin improves emotion recognition for youth with autism spectrum disorders. Biol Psychiatry 67:692694. doi:10.1016/j.biopsych.2009.09.020

Heim C, Young LJ, Newport DJ, Mletzko T, Miller AH, Nemeroff CB (2009) Lower CSF oxytocin concentrations in women with a history of childhood abuse. Mol Psychiatry 14:954-958. doi:10.1038/mp. 2008.112

Hoge EA, Pollack MH, Kaufman RE, Zak PJ, Simon NM (2008) Oxytocin levels in social anxiety disorder. CNS Neurosci Ther 14: 165-170. doi:10.1111/j.1755-5949.2008.00051.x

Hollander E, Novotny S, Hanratty M, Yaffe R, DeCaria CM, Aronowitz BR, Mosovich S (2003) Oxytocin infusion reduces repetitive behaviors in adults with autistic and Asperger's disorders. Neuropsychopharmacology 28:193-198

Hollander E, Bartz J, Chaplin W, Phillips A, Sumner J, Soorya L, Anagnostou E, Wasserman S (2007) Oxytocin increases retention of social cognition in autism. Biol Psychiatry 61:498-503

Jansen LM, Gispen-de Wied CC, Wiegant VM, Westenberg HG, Lahuis BE, van Engeland H (2006) Autonomic and neuroendocrine responses to a psychosocial stressor in adults with autistic spectrum disorder. J Autism Dev Disord 36:891-899. doi:10.1007/s10803006-0124-z

Kagerbauer SM, Martin J, Schuster T, Blobner M, Kochs EF, Landgraf R (2013) Plasma oxytocin and vasopressin do not predict neuropeptide concentrations in human cerebrospinal fluid. J Neuroendocrinol 25: 668-673. doi:10.1111/jne.12038

Lang PJ, Bradley MM, Cuthbert BN (2008) International Affective Picture System (IAPS): Affective ratings of pictures and instruction manual. A-8

Leng G, Ludwig M (2016) Intranasal oxytocin: myths and delusions. Biol Psychiatry 79:243-250 
Lord C, Risi S, Lambrecht L, Cook EH, Leventhal BL, DiLavore PC, Pickles A, Rutter M (2000) The autism diagnostic observation schedule-generic: a standard measure of social communication deficits associated with the spectrum of autism. J Autism Dev Disord 30:205-223

Luteijn F, Barelds DPH (2005) Herziening van de GIT. Handleiding bij de GIT-2. Harcourt Publishers, Amsterdam

Marazziti D, Dell'Osso B, Baroni S, Mungai F, Catena M, Rucci P, Albanese F, Giannaccini G, Betti L, Fabbrini L, Italiani P, Del Debbio A, Lucacchini A, Dell'Osso L (2006) A relationship between oxytocin and anxiety of romantic attachment. Clin Pract Epidemiol Ment Health 2:28

Modahl C, Green L, Fein D, Morris M, Waterhouse L, Feinstein C, Levin $\mathrm{H}$ (1998) Plasma oxytocin levels in autistic children. Biol Psychiatry 43:270-277. doi:10.1016/S0006-3223(97)00439-3

Modi ME, Connor-Stroud F, Landgraf R, Young LJ, Parr LA (2014) Aerosolized oxytocine increases cerebrospinal fluid oxytocin in rhesus macaques. Psychoneuroendocrinology 45:49-57. doi:10. 1016/j.psyneuen.2014.02.011

Neumann ID, Maloumby R, Beiderbeck DI, Lukas M, Landgraf R (2013) Increased brain and plasma oxytocin after nasal and peripheral administration in rats and mice. Psychoneuroendocrinology 38:19851993. doi:10.1016/j.psyneuen.2013.03.003

Onaka T (2004) Neural pathways controlling central and peripheral oxytocin release during stress. J Neuroendocrinol 16:308-312. doi:10. 1111/j.0953-8194.2004.01186.x

Proverbio AM, Adorni R, Zani A, Trestianu L (2009) Sex differences in the brain response to affective scenes with or without humans. Neuropsychologia 47:2374-2388
Rubin LH, Carter CS, Drogos L, Pournajafi-Nazarloo H, Sweeney JA, Maki PM (2010) Peripheral oxytocin is associated with reduced symptom severity in schizophrenia. Schizophr Res 124:13-21. doi:10.1016/j.schres.2010.09.014

Shamai-Tsoory S (2011) The neural bases for empathy. Neuroscientist 17: $18-24$

Spielberger CD (1973) Manual for the state-trait anxiety test for children. Consulting Psychological Press, Palo Alto

Skrundz M, Bolten M, Nast I, Hellhammer DH, Meinlschmidt G (2011) Plasma oxytocin concentration during pregnancy is associated with development of postpartum depression. Neuropsychopharmacology 36:1886-1893. doi:10.1038/npp.2011.74

Strathearn L, Fonagy P, Amico J, Montague PR (2009) Adult attachment predicts maternal brain and oxytocin response to infant cues. Neuropsychopharmacology 34:2655-2666. doi:10.1038/npp.2009.103

Takagi T, Tanizawa O, Otsuki Y, Sugita N, Haruta M, Yamaji K (1985) Oxytocin in the cerebrospinal fluid and plasma of pregnant and nonpregnant subjects. Horm Metab Res 17:308-310. doi:10.1055/ s-2007-1013526

Winslow Jt, Noble PL, Lyons CK, Sterk SM, Insel TR (2003) Rearing effects on cerebrospinal fluid oxytocin concentration and social buffereing in rhesus monkeys. Neuroparmacology 28:910-918. doi:10.1038/sj.npp.1300128

Yuen KW, Garner JP, Carson DS, Keller J, Lembke A, Hyde SA, Kenna HA, Tennakoon L, Schatzberg AF, Parker KJ (2014) Plasma oxytocin concentrations are lower in depressed vs. healthy control women and are independent of cortisol. J Psychiatr Res 51:30-36. doi:10. 1016/j.jpsychires.2013.12.012 